\title{
GRANITE, A NEW VERY HIGH ENERGY GAMAA-RAY TELESCOPE
} C.W.Akerlof ${ }^{1}$, M.F.Cawley ${ }^{2}$, D.J.Fegan ${ }^{3}$, A.M.Hillas ${ }^{4}$, R.C.Lamb
T.C. Weekes

1 Randall Laboratory of Physics, University of Michigan, Ann Arbor, Michigan 48109

2 Physics Department, St. Fatrick's College, Maynooth, Co. Kildare, Ireland.

3 Physics Department, University College, Dublin, 4. Ireland.

4 Fhysics Department, University of Leeds, Leeds, United Kingdom

5 Physics Department, Iowa State University, Ames, Iowa. 50011.

6 Fred Lawrence Whipple Observatory, Harvard-Smithsonian Center for Astrophysics, P.0.Box 97, Amado, Arizona 85645

A international collaboration of astrophysicists plan to construct a new 10-meter diameter gamma-ray Cerenkov telescope at the Whipple Observatory on Mt. Hopkins in Arizona. By operatin in coincidence with the original 10 -meter reflector, the sensitivity for gamma-ray detection will improve by more than an order of magnitude. The new telescope, named GRANITE (for GammaRay Astrophysics New Imaging Telescope), will include a 109-element imaging photon detector wit a $0.2^{\circ}$ pixel size. The entire system consists of a steerable alt-azimuth mount, a faceted mirror asserably, a highly segmented photon detector, and ancillary data acquisition and control electronic:s. This telescope is considered an engineering prototype for a large array of Cherenkov detectors which will further enhance our ability to detect astrophysical sources of very high energy gamma rays.

\section{INTRODUCTION}

The development of astronomy during the last three decades has been strongly supported by new instrumentation covering electromagnetic frequencies that were previously unobservable. The last bands of the electromagnetic spectrum still awaiting this kind of development are at very high energy. A significant increase in our knowledge of the gamma-ray sky at lower energies will begin next year when the GammaRay Observatory (GRO) is launched by NASA.

Although the EGRET detector onboard the GRO will boast an order of magnitude increase in sensitivity over its predecessors, it will detect few quanta more energetic than $10 \mathrm{GeV}$ for lack of sufficient flux. For the past 30 years, experimental groups have been trying to circumvent the size limitations of satelliteborne detectors by looking for gamma-ray interactions in the atmosphere using groundbased instruments. Two techniques have been pursued; detection of Cherenkov light from showers with energies greater than $100 \mathrm{GeV}$ and detection of charged particle secondaries by large scintillation counter arrays for energies greater than $100 \mathrm{TeV}$. Both methods attain sensitivity by being able to sense showers over an area of the order of $3 \times 10^{4} \mathrm{~m}^{2}$. Unfortunately, the background of hadron showers is so overwhelming that many claims of gammaray detection above $100 \mathrm{GeV}$ have not been verified. These first generation systems do not distinguish the gamma ray showers from the much more numerous hadronic background.

\section{CHERENROV IMAGING TECHIIQUE}

Progress in identifying gamma-ray sources must begin with the rejection of the hadronic background. There are sufficient differences between the pure electromagnetic cascade and the hadronic showers to permit this distinction with both techniques. The shower characteristics that can be used are listed in Table 1; to date only the Cherenkov imaging technique has been successfully used to detect a cosmic source ${ }^{1}$. At PeV energies the muon-toelectron ratio is potentially a powerful technique but to date discrimination based on showe: age seems to be more useful ${ }^{2}$. Shower simulation studies do not predict age to be a useful kind of discrimination.

The Cherenkoy imaging technique was first 
proposed using image intensfier systems ${ }^{3}$; practical considerations prevented its development along these lines. In 1978 a more practical approach was proposed using arrays of phototubes in the focal plane of large reflectors ${ }^{4}$. The first such system 5 was developed for the thipple Observatory $10 \mathrm{~m}$ Optical Reflector which had been used in very high energy gamma-ray astronomy since 1968. The first phase of this development involved a collaboration between the Smithsonian, Iowa State University, the University of Hawail, University College, Dublin, and the University of Durham. Between 1982 and 1985 a 37-element camera was built and used to observe a variety of sources $^{6}$.

A key point in the development of the technique was the publication of simulations 7 which predicted that the technique was capable of even greater discrimination than had been previously considered. This discrimination arises from the improved angular resolution for the detection of a point source and from the differences in the transverse width of electromagnetic and hadronic showers. These predictions were verified in the detection of the Crab Nebula with a statistical significance which is not normally encountered in very high energy gamma-ray astronomy (nine sigma) ${ }^{l}$. A second generation system with 109 pixels $\left(0.25^{\circ}\right.$ spacing), the so-called High Resolution Camera, was commissioned in 1988 and resulted in a significant improvement in flux sensitivity; these observations yielded a 15 sigma detection of the Crab Nebula which has been reported at this workshop ${ }^{8}$. Hopefully a detection at this level is sufficient to convince those who have been skeptical (Derhaps rightfully) of many of the earlier claims at source detections.

The thipple Collaboration detection has been Independently confirmed by the University of Michigan group ${ }^{9}$ who used two $11 \mathrm{~m}$ reflectors at Sandia Labs in Albuquerque; this system rejected showers with a simple imaging classification. These two groups are now collaborating to build a new generation of air Cherenkov detectors which are expected to have an order of magnitude more sensitivity than any now in existence. The prototype instrument, called GRANITE, will be constructed on Mt. Hopkins, Arizona with completion possible in late 1990.

\section{OPTICAI DESIGN PARAMETERS}

As in the original camera the design of the reflector results from Monte Carlo simulations of the signals expected from gammarays in the energy region of interest. These simulations' have already been completed for the High Resolution Camera and will be extended to include the addition of a second camera. The simulations predict the Cherenkov light distribution modified by the optical aberrations in the reflector and the effect of background noise fluctuations. The light images expected from an isotropic distribution of hadron showers is also simulated and used to compare with the measured shower light image background.

The optical distribution pattern of air shower Cherenkov light tightly constrain the design of the optimal detector. An essential goal of the GRANITE detector is operation at the lowest possible threshold in order to Improve hadronic rejection and enhance sensitivity. Experience has shown that the optical concentrator collecting area must exceed $75 \mathrm{~m}^{2}$ to obtain a threshold as low as $100 \mathrm{GeV}$ The fast signal risetimes necessitate a parabolic reflector surface to provide isochronous paths for all Cerenkov light rays.

The inherent large size of the optical concentrator invites a faceted mirror design similar to that used at the Whipple Observatory and to several developed for solar thermal energy applications. The optical accuracy 
requirements are at least a factor of two more stringent than for solar energy whose source has an apparent diameter of $0.5^{\circ}$. To obtain adequate image sharpness over the full angular acceptance of 2 millisteradians, the focal length to aperture ratio must be greater than 1.0. This also reduces the aberrations introduced by using spherical facets to approximate a paraboloidal surface. Ray trace plots for rays parallel to the optic axis and $1.5^{\circ}$ from the axis are shown in figure 1 for our current design. The calculated spot size is $0.07^{\circ} \times 0.15^{\circ}$ FWH for the extreme case. A recent study 10 indicates that in principle the Cherenkov light images have directional

information on a scale of 2 arc-min; this kind of resolution is superior to that available even in satellite experiments.
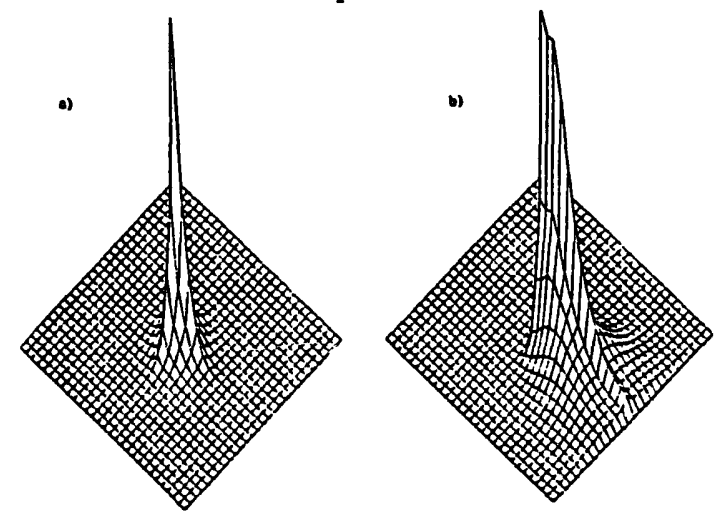

Figure 1. Optical images of a point source. The grid subtends $1^{\circ}$ by $1^{\circ}$. (a) on-axis (b) off-axis

\section{SITE}

The GRANITE detector will be constructed on the southern ridge of $\mathrm{kt}$. Hopkins near Amado, Arizona at a latitude of $31^{\circ} 41^{\circ}$ and an altitude of 2300 meters. This is the site of the Whipple Observatory and is the home of a variety of optical telescopes including the MMT. Twenty years of site experience show that this is one of the premier sites for optical observing in the U.S.A. The ridge is shared by the existing Whipple 10 meter Gamma Ray Reflector and the 61 and $150 \mathrm{~cm}$ optical telescopes. The spacing between the gama-ray telescopes will be 120 meters to permit the stereosiopic reconstruction of each cosmic ray event. Figure 2 shows a sketch of the area viewed from the sast.

\section{MECHANICAI STRUCTURE}

The optical concentrator mechanical structure will be modelled on the existing 10meter Whipple reflector which has operated reliably for over 20 years. The mount is an elevation over azimuth design with the azimuth rotation supported by a kingpost. The new device shares many of the requirements of large satellite communications dishes and solar thermal parabolic concentrators. For this reason, we intend to take advantage of extensive commercial experience in fabricating these kinds of structures. Satellite dishes operate routinely in remote locations under a

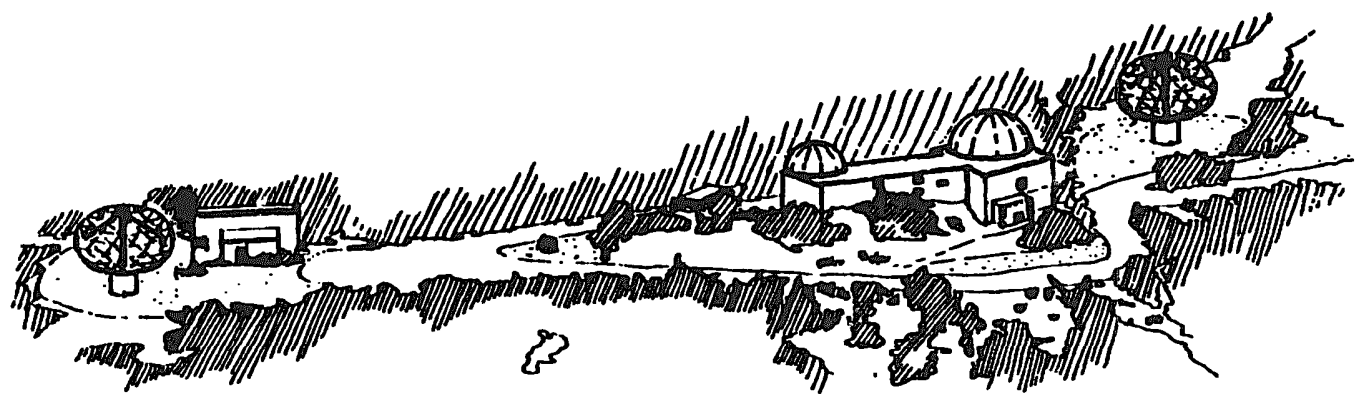

Figure 2. The 7,600 foot ridge of Mount Hopkins. The existing $10 \mathrm{~m}$ reflector is on the left, the proposed one is on the right. 
variety of extreme conditions with little maintenance. The microwave optics of these antennae is typically a Cassegrainian configuration with an $f$ number of 0.3 so that an off-the-shelf unit is not entirely compatible with our need for longer focal lengths. Various large antenna fabricators have expressed an interest in tailoring a structure that would meet our specifications. A sketch of one such example is shown in figure 3. This is very similar to the design of the

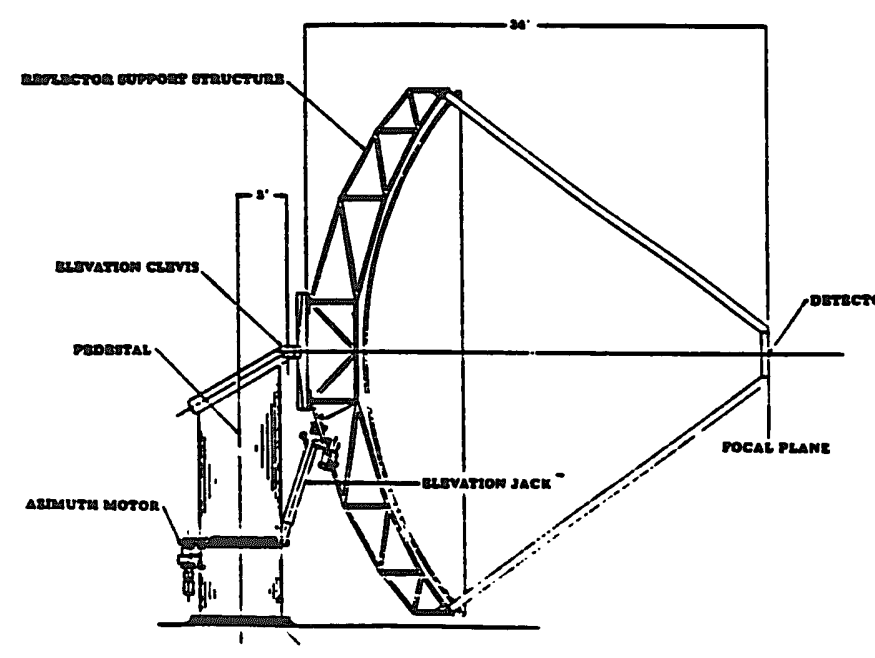

Figure 3. Stetch of possible configuration of new reflector mount seen from the side.

Whipple Observatory $10 \mathrm{~m}$ reflector which is well suited to this work but which is expensive to duplicate exactly. Solar technology does not currently offer a competitive system.

Tracking control systems for satellite dishes can provide tracking accuracies of $0.05^{\circ}$ or better which is quite suitable for our application. We will encourage the vendor to provide the software capability of specifying the target right ascension and declination so that the conversion to azimuth and elevation can be continually updated internally by the controller microprocessor.

One of the critical components of the GRANITE detector is the array of mirrors that focus the Cherenkov light on the photomultiplier camera assembly. The low light levels require that the reflectivity be high, especially in the 330-380 nanometer wavelength region which spans the maximum sensitivity band for Cerenkov radiation. The mirrors will be continuously exposed to the environment so their reflectivity must be protected from degradation over an extended period. In addition, since image resolution is critical for hadron background rejection, the mirrors must provide an adequately sharp focus. To satisfy these requirements at a reasonable cost, we have chosen to copy the general method developed by Maurice Argoud for the mirrors installed on the Test Bed Concentrators now at Sandia National Laboratories. These are second-surface mirrors bonded to foam glass blocks and sealed against moisture. Their rugged character has been proven over many years of outdoor exposure.

The mirror curvature is formed by stressing $1 \mathrm{~mm}$ glass sheet to a spherical contour with a convex aluminum plate machined to the desired radius. Only a few pounds of force are required. The shape is maintained by epoxying the opposite, aluminized side of the glass sheet to a 2" thick foam glass block with a matching shallow concave depression. The glass foam provides a stiff support that will not creep or distort with time. The mirror facets are hexagonal in shape with a width of 18 " across the flats. An accuracy of 1.0 milliradians has been obtained for prototype facets; this error must be held less than half the desired angular resolution. 421 facets will be required for a 10 meter diameter concentrator.

This method has several advantages nver more conventional techniques. Since the mirror is second-surface, dust and dirt can be easily removed by scrubbing with water and detergent with no fear of damaging the reflective coating. The mirror facets are light in 
weight, placing fewer demands on the supporting mechanical structure. Finally, these mirrors require no high temperature processing (such as glass slumping) so the manufacture can be done easily and cheaply with a minimum of equipment. The major cost will be vacuum aluminization of the mirror glass.

\section{PHOTOMULTIPLIER CAMERA}

The Cherenkov photon detector will be constructed from an array of 91 1-1/8" diameter photomultipliers surrounded by an additional 18 2" tubes. The pixel spacing corresponds to 3.2 milliradians which is a good match to the angular width of electromagnetic shower images. This design is a carbon copy of the detector head in the High Resolution Camera on the Whipple Observatory $10 \mathrm{~m}$ Reflector. A example of an event recorded by this camera is shown in figure 4. This camera has already been demonstrated to be a factor of ten better than a non-imaging system 8 . The risetime for the inner tubes is 2.2 nanoseconds and 568 of the central area will be covered by photocathode. A signal of 80 photoelectrons is expected from a $100 \mathrm{GeV}$ gamma-ray shower.

\section{DATA ACQUISITION}

The data acquisition system must fulfill a number of demanding requirements. Briefly, these are:

1. minimal perturbation of the existing 10 meter telescope data acquisition system;a full observing program is planned during the construction of the second camera which will coincide with the first months of operation of the GRO mission;

2. the ability to record events with burst rates to $1 \mathrm{kHz}$;

3. capability of measuring both the pulse height and time structure of the Cherenkov light pattern;

4. capability of operating in a stand-alone

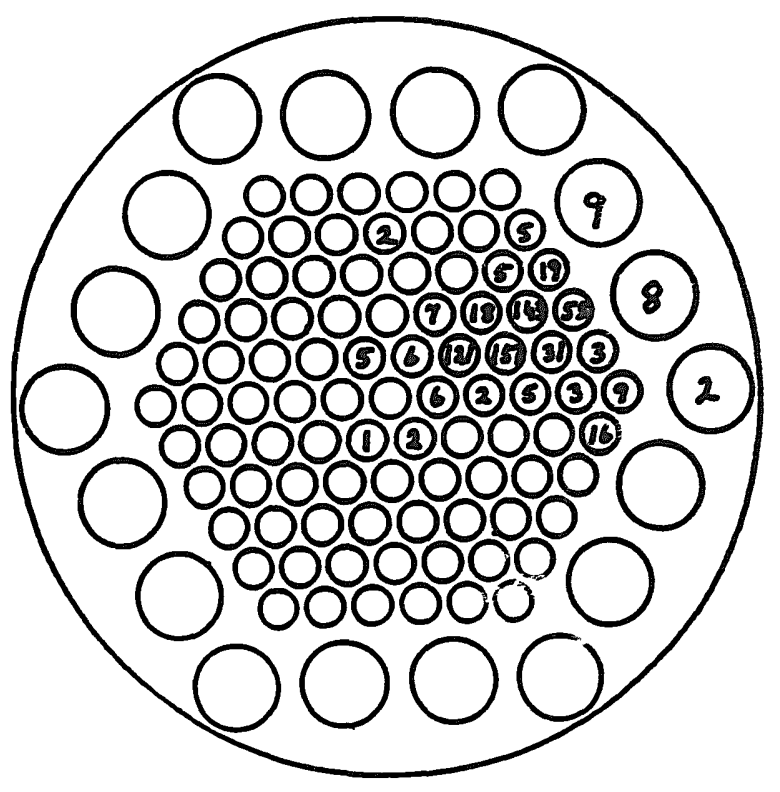

Figure 4. Layout of phototubes in Whipple Observatory HRC with event superimposed (numbers are photoelectrons).

mode, independent of the status of the remote telescope.

These considerations have led us to adopt a two-level trigger logic that accepts a relatively weak signal in time coincidence with a signal from the remote dish or a larger amplitude signal from the local phototube array with no corroborating pulse from the remote instrument. The large physical separation of the two reflector ${ }^{-} 120$ meters) imposes an additional constraint on the logic design. With typical time differences of $200 \mathrm{~ns}$ between signals in the two telescopes, analog signals must be captured long before possible coincidence information can arrive from the distant tube array. The trigger logic will be based on the multilevel architectures of modern high energy physics experiments. A primary trigger rate of up to $10 \mathrm{kHz}$ will be formed by a minimal requirement that two or more phototube pulses exceed threshold within a 20 ns resolving time. This primary trigger will enable all ADC and TDC gates for the local array and initiate a logic timing pulse to the 
remote array. If the particular event exceeds a more stringent, higher threshold, the analog signals will be converted and data transmitted to the local data acquisition computer with no further intervention. For events that do not meet this criterion, a clear pulse will be generated if a remote trigger signal is not received within a narrow tiane window whose position varies with the instantaneous sky position of the tracked star. To properly identify those events that have data recorded in both arrays, clock registers raust be installed in both systems to provide time stamps with a resolving time of $20 \mathrm{~ns}$.

A large fraction of the required electronics is available commercially. The overall data acquisition system will be configured around several CAMAC crates which house the analog modules that measure the photomultiplier signals.

The general philosophy of the GRANITE detector is to extract as much information as possible from each shower event. For each photomultiplier pulse, the pulse height, width, and arrival time will be separately measured. The trigger rate for each tube will be scaled and a monicor test point will be available to display and digitize the entire pulse waveform for selected channels. To provide for this large signal fanout, an 8-channel amplifierdiscriminator module has been designed to incorporate a wide band amplifier and two independent discriminators. The discriminator outputs feed the coincidence logic, the TDCs, and rate sca?ers. Two externally supplied reference voltages control the discriminator thresholds.

The projected event rate is approximately 50 $\mathrm{Hz}$ for an energy threshold of $100 \mathrm{GeV}$. The resulting data stream of 10 kilobytes per second will generate 300 Mbytes for a full night of running, equivalent to two full magnetic tapes at a density of 6250 BPI.

\section{EXPECTED PERFORMANCE}

We estimate that the combined effect of improved imaging and lowered energy threshold will permit a factor of 12 increase in sensitivity for showers imaged in both Cherenkov telescopes. This corresponds to a factor of 3.5 in distance so that an object similar in luminosity to the Crab Nebula could be reliably detected at a distance of 7

kiloparsecs. We expect the total number of visible objects to increase by an order of magnitude. The incident photon direction will be determined to a precision of 0.1 degrees or better. This accuracy is as good or better than any other HE, VHE or UHE gamma-ray detector either planned or now in existence.

The stereo imaging information will completely specify the photon direction and ground impact point. This is critical for accurately defining a sensitive fiducial area for computing absolute flux levels and to permit the measurement of the energy spectrum. Since the telescope aperture is much wider than the shower image, it will also be possible to record showers from a target star simultaneously with off-angle hadronic showers which determine the background.

The GRANITE detector has been designed as a prototype for a larger array of telescopes. By combining imaging information from many dishes which blanket the Cherenkov light pool, we hope to reduce the hadronic background even further. Our collaboration looks forward to the next few years to see what mysteries will be revealed in this newly opened window of the electromagnetic spectrum.

\section{ACKNOWLEDGEMENTS}

The development of the imaging technique was made possible by the contributions of all former and present members of the Whipple Observatory Collaboration. Our knowledge and understanding of large parabolic antennae and 
solar concentrators has benefitted from many conversations with $C$. Cameron, V. Goldberg, and M. Quintana. This work was supported by U. S. Department of Energy.

\section{REFERENCES}

1. Weekes, T.C. et al. (1989) Ap. J., (to be published), July 1, 1989.

2. Weekes, T.C. (1987), Phys. Rep. 160, 1.

3. Jelley, J.V. and Porter, N.A., 1963, Quart.

J. Astron. Soc., 275.

4. Weekes, T.C. and Turver, K.E., (1977) in Proc. 12th ESLAB Symposium, Frascati, ESA SP124, 279.
5. Weekes, T.C., 1981, Proc. 17th I.C.R.C., Paris, 8,34 .

6. Cawley, M.F. et al. 1985, Proc. 19th

I.C.R.C., La Jolla, 1, 131.

7. Hillas, A.M., 1985, Proc. 19th I.C.R.C., La Jolla, 3, 445.

8. Lang, M.J. et al. (1989), this workshop.

9. Akerlof, C.A. et al. (1989) Proc. of the Gamma-Ray Observatory Workshop, (to be published), NASA/Goddard SFC, Greenbelt, MD. 10.Hillas, A.M. (1989), Proceedings of the Crimean Workshop on VHE Gamma-Ray Astronomy, (to be published), April 1989. 